\title{
The Effect of Nasopharyngeal Ventilation on the Detection Rate of Colorectal Polyps in Two Hundred Middle-Aged and Elderly Overweight Patients
}

\author{
Zhaomeng Zhuang ${ }^{1}$, Juanhong Zheng ${ }^{1}$, Min Xie $^{1}$, and Shuo Chen ${ }^{1}$ \\ ${ }^{1}$ Wenzhou Integrated Traditional Chinese and Western Medicine Hospital
}

April 22, 2021

\begin{abstract}
Objective To analyze the effect of nasopharyngeal ventilation on the detection rate of colorectal polyps in 200 middle-aged and elderly overweight patients during painless colonoscopy. Methods A total of 100 patients aged 50-75 years, body mass index (BMI) [?]24 or abdominal circumference [?] $85 \mathrm{~cm}$ in males and [?] $82 \mathrm{~cm}$ in females, without underlying diseases, who underwent nasopharyngeal ventilation during painless colonoscopy at physical examinations in our hospital from 2019 to 2020 , were selected as the observation subjects $(\mathrm{n}=100)$. The control sample area was determined by propensity matching according to the basic information characteristics presented by the observation group. 100 patients received mask oxygen at physical examinations were randomly selected in the control group $(\mathrm{n}=100)$. Results The verification analysis after matching indicated that there were no intraoperative and postoperative adverse reactions, the number of intraoperative limb movements was less than 1 , and the intestinal peristalsis intervals were more than $5 \mathrm{~s}$ in both groups, without atropine intervention. Colonoscopy was performed by senior endoscopists with a withdrawal time of 6-8 min. When the intraoperative SpO2 was lower than $90 \%$ but higher than $85 \%$, the patients in the observation group underwent nasopharyngeal ventilation while those in the control group received mask oxygen assisted chin-lift. As a result, the SpO2 was maintained higher than $90 \%$. The detection rate of colorectal polyps was compared between the two groups. Conclusion The detection rate of colorectal polyps in the observation group was higher than that in the control group, with a statistically significant difference. It may result from the reduction of ineffective respiratory movement and intraoperative intestinal peristalsis in middle-aged and elderly overweight patients.
\end{abstract}

\section{Hosted file}

Nasopharynx.pdf available at https://authorea.com/users/409456/articles/519134-the-effectof-nasopharyngeal-ventilation-on-the-detection-rate-of-colorectal-polyps-in-two-hundredmiddle-aged-and-elderly-overweight-patients 